\title{
Multimodal Computed Tomography in Acute Ischemic Stroke
}

\author{
Sachin Rastogi, MD ${ }^{1}$ and David S Liebeskind, MD, FAHA ${ }^{2}$ \\ 1. Fellow, Neuro-interventional Radiology; 2. Associate Professor of Neurology, University of California, Los Angeles
}

\begin{abstract}
Stroke is the third leading cause of death in the US, affecting 795,000 individuals annually. Currently, only a small percentage of acute stroke patients receive thrombolytic treatment. A significant limitation is the current use of strict time criteria in the decision to treat. As there are significant interindividual variations in response to an acute vascular occlusion, the goal of modern imaging such as multimodal computed tomography (CT) is to rapidly identify acute ischemic stroke patients and determine which patients are likely to benefit from treatment based on tissue perfusion status rather than time of presentation alone. Multimodal CT consists of a non-contrast head CT, CT angiogram (CTA) of the head and neck, and CT perfusion (CTP). The non-contrast head CT allows rapid triage of a patient with hemorrhagic versus ischemic stroke. The CTA allows identification of the site of vascular pathology with similar quality to digital subtraction angiography. The CTP scan allows for determination of the infarct core and surrounding ischemic penumbra, which remains at risk for infarction if perfusion is not restored. This allows the potential to prospectively treat only those patients likely to benefit from thrombolysis while protecting those patients unlikely to benefit from the risks associated with treatment.
\end{abstract}

\section{Keywords}

Stroke, multimodal computed tomography (CT), non-contrast head CT, CT angiography, CT perfusion, ischemic penumbra

Disclosure: Sachin Rastogi, MD, has no conflicts of interest to declare. David S Liebeskind, MD, FAHA, is funded by National Institutes of Health (NIH)/National Institute of Neurological Disorders and Stroke (NINDS) grants K23NS054084 and P50NS044378.

Received: May 15, 2010 Accepted: June 28, 2010 Citation: US Neurology, 2010;6(1):50-4 DOI: 10.17925/USN.2010.06.01.50

Correspondence: David S Liebeskind, MD, FAHA, 710 Westwood Plaza, LoS Angeles, CA 90095. E: davidliebeskind@yahoo.com

Stroke is the third leading cause of death in the US, affecting approximately 795,000 patients annually. ${ }^{1}$ A recent analysis shows that only $3-5 \%$ of acute stroke patients actually receive intravenous thrombolytic therapy. ${ }^{2}$ An important cause of non-treatment is the strict time criteria for acute medical and interventional therapies: many acute stroke patients are ineligible for treatment due to uncertainty in time of onset or delayed presentation.

The use of strict time criteria to make treatment decisions is not ideal since there are significant differences among individuals and their responses to an acute vascular event. ${ }^{3}$ The goal of advanced neuroimaging is to individualize acute stroke treatments based on assessment of tissue viability and vascular status rather than using strict time criteria alone. Acute intervention or treatment may be refined through improved selection of subjects likely to benefit balanced by the risk of potential harm, irrespective of the time of presentation. ${ }^{3}$ Multimodal computed tomography (CT), including noncontrast CT (NCT), CT angiography (CTA), and CT perfusion (CTP), is increasingly available and may serve as an ideal tool for rapid image evaluation and triage of the stroke patient.

The goal of a multimodal CT protocol in acute stroke is to answer four key questions: ${ }^{4}$
- Is there hemorrhage?

- Is there occlusion of a proximal artery or intravascular thrombus that can be targeted for thrombolysis?

- Is there a core of critically ischemic irreversibly infarcted tissue?

- Is there a penumbra of ischemic but potentially salvageable tissue?

The first question is readily addressed by NCT and the second question is addressed by CTA. The last two questions are the most challenging and may potentially be addressed by CTP. ${ }^{5}$

\section{Technique}

The first component of the multimodal CT acquisition is the noncontrast head CT. Axial images through the brain are obtained in a plane parallel to a line drawn from the orbital floor to the external auditory meatus using $120 \mathrm{kVp}, 300 \mathrm{~mA}$, and a $23 \mathrm{~cm}$ field of view. Next, the perfusion scan is performed with injection of $50 \mathrm{ml}$ of intravenous iso-osmolar non-ionic iodinated contrast at $5 \mathrm{ml} / \mathrm{second}$. The use of iso-osmolar contrast agents as opposed to low-osmolar contrast agents is preferred in order to minimize nephrotoxity. ${ }^{6,7}$ This is important because the patient's baseline renal function may not be available at the time of the emergent scan and there may be potential for the patient to receive additional iodinated contrast if endovascular intervention is needed. With our current 64-channel CT scanners, 
perfusion imaging is performed at six axial $4.8 \mathrm{~mm}$-thick slices centered above and below the caudate head. Images are obtained with 80kVP and 120mA for 45 seconds. Finally, a CTA of the brain and neck is performed with intravenous injection of $100 \mathrm{ml}$ of contrast at $3 \mathrm{ml} /$ second utilizing a $120 \mathrm{kV}$ and $300 \mathrm{~mA}$ technique and bolus tracking on the ascending aorta with a scan trigger of 150 Hounsfield units. Axial $0.625 \mathrm{~mm}$ slices are obtained from the thoracic inlet to the skull vertex. The CTP and CTA images are post-processed on a separate networked workstation. On average, image acquisition, processing, and interpretation can be performed in 15 minutes. ${ }^{8}$

\section{Non-contrast Computed Tomography}

The non-contrast head CT comprises the first set of images obtained in the acquisition of multimodal CT imaging. The primary purpose of the NCT is identification of hemorrhagic stroke, which accounts for approximately $20 \%$ of all acute strokes. ${ }^{9}$ Stroke mimics such as tumor, infection, or inflammatory causes may also be detected on the noncontrast head CT. Important information regarding parenchymal changes associated with hyper-acute ischemic stroke may also be detected from the non-contrast study. In the less-than-six-hour time window from symptom onset, signs of early infarction can be seen in approximately $61-85 \%$ of patients..$^{10,11}$ The appropriate use of window (W) and level ( $L$ ) settings is required to detect the subtle findings associated with early ischemic changes. It is recommended that images are reviewed with both standard brain window and level settings of $40 \mathrm{HU} W / 2 \mathrm{OHU} L$ followed by repeat evaluation with a narrower setting of $20 \mathrm{HU} \mathrm{W} / 32 \mathrm{HU} \mathrm{L}^{12}$ Findings that have been associated with early ischemic change on CT include subtle hypoattenuation, obscuration and loss of gray matter-white matter differentiation, cortical sulcal effacement, loss of the insular ribbon, and obscuration of the lentiform nucleus. Concomitant findings in the vessels may include hyper-attenuation of a large vessel such as 'hyperdense middle cerebral artery (MCA) sign' or 'dot sign' in an M2 branch $^{13-15}$ (see Figure 1).

These early CT findings in acute ischemic stroke are helpful not only for confirming the diagnosis of acute ischemia but also useful for predicting patient outcome and response to thrombolytic therapy. ${ }^{16-20}$ For example, the hyperdense MCA sign in acute stroke patients has been associated with poor outcomes and, in some studies, failure to recanalize with intravenous (IV) thrombolytics. ${ }^{14,21}$ The extent of early ischemic change in the MCA territory also plays an important role in patient prognosis and safety of IV thrombolytics. Studies show an increased risk for symptomatic intracranial hemorrhage with IV thrombolytics when more than one-third of the MCA territory demonstrates ischemic change on an NCT. ${ }^{17,19,20,22,23}$ In order to more reliably quantify the extent of early ischemic abnormality, the Alberta Stroke Program Early CT Score was designed for the MCA territory. This system divides the MCA territory into 10 regions: caudate head, lentiform nucleus, insular ribbon, internal capsule, and anterior, middle, and posterior, superior, and inferior MCA territories. In this system a score of 10 is considered normal. A score of 7 or less indicates the presence of extensive early ischemic change and thus the potential for increased risk for hemorrhagic transformation. ${ }^{24}$

Other findings on NCT may provide useful insight into a patient's medical history. This is especially important in the acute setting since

\section{Figure 1: Early Infarct Signs}

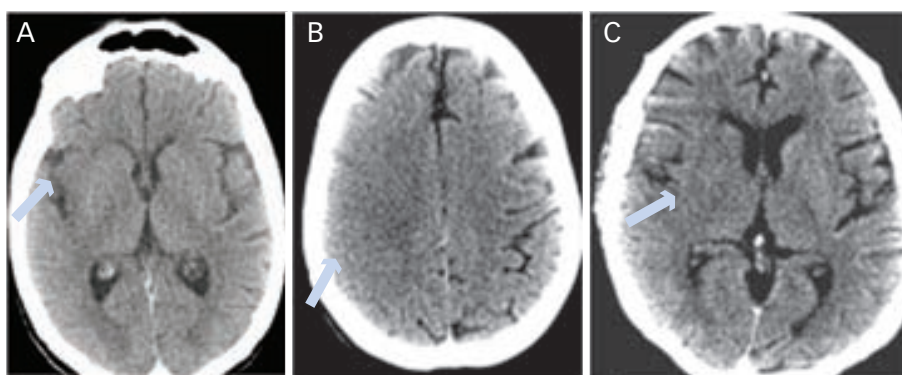

A: Loss of gray-white differentiation of the right insular ribbon. B: Sulcal effacement and subtle loss of gray-white differentiation in the right middle cerebral artery territory. C: Obscuration of the right caudate head and lentiform nucleus.

\section{Figure 2: Computed Tomography Angiogram Demonstrating an M2 Occlusion}
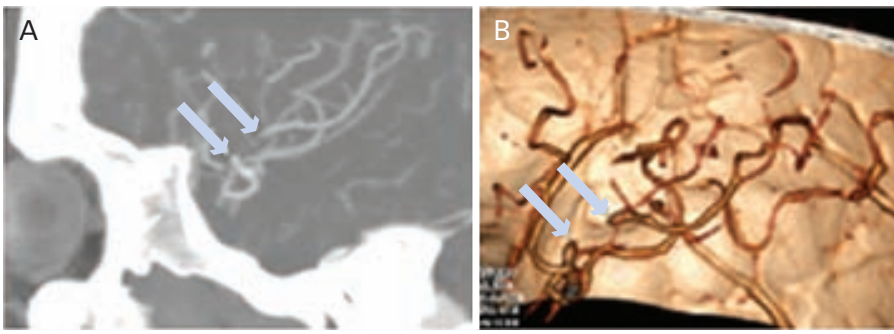

Collateral flow allows for visualization of the segmental middle cerebral artery branch distal to the occlusion, making detection of the lesion more challenging without the use of (A) sagittal thick maximum-intensity projection and (B) 3D shaded surface volume rendering.

obtaining a patient's past medical history is often difficult. From the non-contrast scans, evaluation for vascular calcifications, lacunar infarcts, and periventricular white-matter hypo-attenuation can be highly suggestive of underlying vascular risk factors such as diabetes, hypertension, hyperlipidemia, chronic renal disease, and/or significant smoking history. ${ }^{25-30}$ Also, the presence of prior cortical infarcts, especially in different vascular territories, will significantly increase the probability of an embolic stroke etiology.

\section{Computed Tomography Angiography}

With current 64-channel multidetector CT (MDCT) technology, rapid high-resolution imaging of the vasculature from the aortic arch to the skull vertex can be performed in about four to six seconds with diagnostic quality near that of conventional digital subtraction angiography (DSA). ${ }^{31,32}$ Compared with DSA, CTA demonstrates a sensitivity and specificity of 98.4 and $98.1 \%$, respectively, for vascular patency of the intracranial arteries from the internal carotid artery to the proximal vessels of the circle of Willis and the sylvian MCA vessels. ${ }^{33}$ The intracranial vessel status depicted by CTA has been shown to have prognostic value in response to IV tissue plasminogen activator (tPA). The presence of proximal MCA occlusion compared with distal MCA occlusion or no occlusion to the M3 level on CTA has been shown to be a predictor of worse clinical outcome in patients receiving IV tPA. ${ }^{34}$ Other studies using CTA have shown that there is little benefit from IV recombinant tPA (rtPA) in patients who demonstrated poor collateral flow by CTA, spontaneous recanalization, or proximal 'top of internal carotid artery (ICA)' saddle emboli. ${ }^{34,35}$ Due to the poor response to IV tPA in patients with proximal 
Figure 3: A Patient with Acute Left Middle Cerebral Artery Territory Stroke
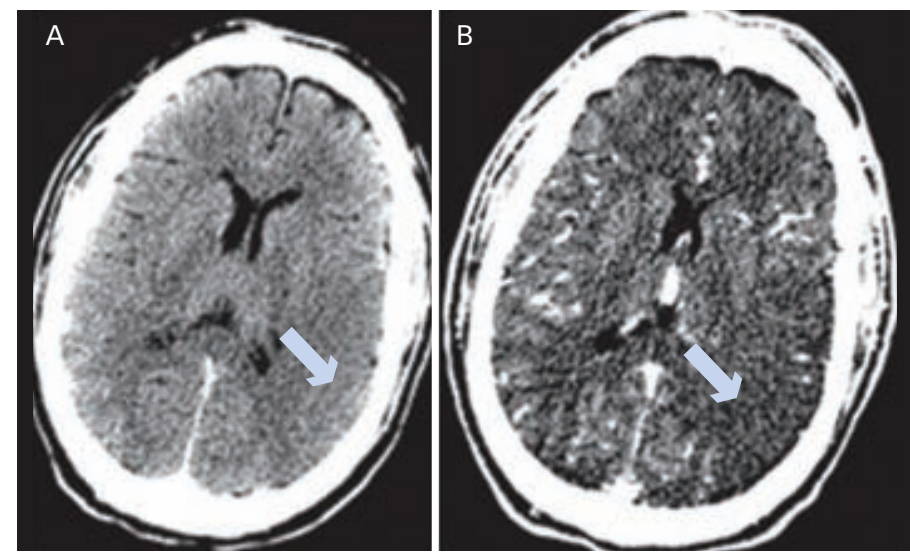

A: Non-contrast computed tomography (CT) demonstrates subtle loss of gray-white differentiation in the left fronto-parietal region. B: CT angiography (CTA) source image (SI) better depicts the acute ischemic change in the left fronto-parietal region.

Figure 4: A Normal Computed Tomography Perfusion Scan
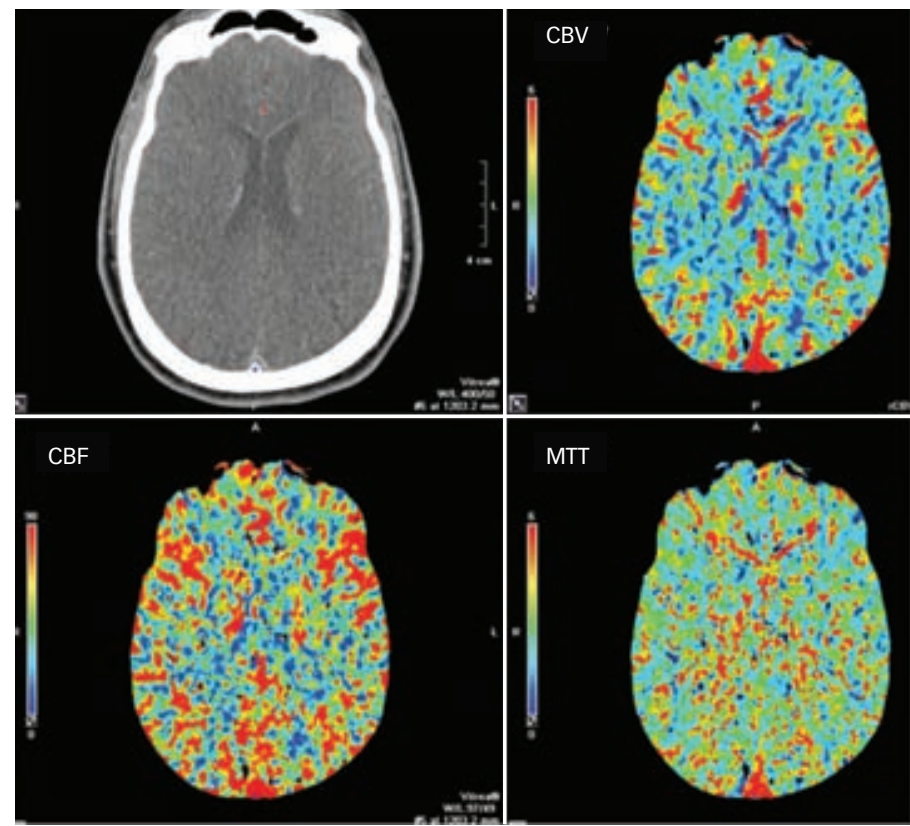

The top right image demonstrates the selection of the arterial input function (red dot) in the A2 segment and the venous output function (blue dot) in the superior sagittal sinus. The normal symmetric cerebral blood volume (CBV), cerebral blood flow (CBF), and mean transit time (MTT) are shown.

intracranial vessel occlusions, some researchers are investigating early intra-arterial (IA) tPA in the less-than-three-hour time window based on CTA. ${ }^{36}$

The neck CTA component also provides important additional information for the management of an acute stroke patient. Carotid atherosclerosis accounts for approximately $30 \%$ of all ischemic strokes. ${ }^{37}$ Carotid stenosis detected by CTA is comparable to that measured by DSA, with a sensitivity of $100 \%$ and specificity of $95 \%$ when the axial source images (SIs) are used. ${ }^{38}$ In addition, CTA provides direct characterization of the atherosclerotic plaque, depicting ulceration, the soft and calcified components, and the thickness of the fibrous cap. ${ }^{39}$
Near-occlusive carotid stenosis, a potential pitfall for carotid Doppler ultrasound, can also be detected by CTA. ${ }^{40}$ CTA of the neck is also capable of depicting cervicocephalic dissections, a common cause of acute stroke in young patients. ${ }^{41,42}$

CTA images should be analyzed in a systematic process given the large size of the data set. In the setting of acute stroke, detection of vessel occlusion is of paramount importance. This is most rapidly accomplished by reviewing the coronal maximum-intensity projection (MIP) re-formatted views that are readily available at the console on most scanners. Large vessel occlusions involving the ICA, intra-dural vertebral arteries, basilar artery, M1, A1, and P1 are readily identified on coronal MIP views. If an abnormality explaining the patient's symptoms is not readily apparent, careful review of the axial SIS, with the use of both 3D and thick MIP re-formatted views, on a dedicated 3D workstation is recommended. A common pitfall in the interpretation of CTA is missing an occlusion because retrograde opacification of the vessels distal to the occlusion via collaterals makes the detection of a focal occlusion challenging (see Figure 2). After identification of the vascular lesion it is important to note the status of the collateral circulation from Willisian and leptomeningeal collaterals.

In addition to evaluating the vasculature, source CTA images provide valuable information about perfusion through the brain parenchyma. This is an underutilized feature of CTA, since most multimodal CT protocols incorporate CTP imaging for a detailed evaluation of the brain parenchymal perfusion. However, CTA SIs may be helpful if the area of concern is not covered in the CTP images, especially with regard to the posterior fossa. CTA SIs have been shown to be superior to NCT for detecting acute ischemic changes of the posterior circulation. ${ }^{43,44}$ Also, the CTA SIs are helpful when the CTP images are limited by patient motion or inappropriate contrast bolus. When interpreting parenchymal changes on CTA SIs, attention has to be paid to the scanner on which they are acquired. With the earlier-generation 16-channel or fewer MDCTs, they have been shown to be more representative of cerebral blood volume and have correlated well with diffusion-weighted imaging (DWI) abnormalities seen on magnetic resonance imaging (MRI). ${ }^{45,46}$ However, with current 64-channel MDCT, due to the rapid image acquisition, lesions seen on CTA SIs more closely approximate cerebral blood flow (CBF) lesion volumes seen on CTP. Regardless of the scanner, CTA SIs are superior to CT in the detection of early ischemic changes, as shown in Figure 3.

\section{Computed Tomography Perfusion}

CTP is the most complex component of multimodal CT for acute stroke. It has been shown to increase sensitivity for diagnosing acute ischemia compared with CT and CTA alone. ${ }^{47}$ In addition, CTP has the possibility of differentiating the infarct core from the ischemic penumbra. A recent study suggests CTP can be used to predict benefit after thrombolysis. ${ }^{48}$

The three main CTP parameters used to evaluate acute stroke patients are cerebral blood volume (CBV), CBF, and mean transit time (MTT). These parameters are related to each other by the central volume principle, which states that $\mathrm{CBV}=\mathrm{CBF} \times \mathrm{MTT}{ }^{49} \mathrm{CBV}$ represents the volume of blood per unit brain mass $(\mathrm{ml}$ per $100 \mathrm{~g}$ of 
brain tissue). MTT represents the time difference from arterial inflow to venous outflow (seconds). CBF represents the volume of blood per minute moving through a unit of brain mass ( $\mathrm{ml} /$ minute per $100 \mathrm{~g}$ of brain tissue). These parameters are calculated from the source data most commonly by using a mathematical process known as delay-insensitive or delay-compensated deconvolution. ${ }^{5,50}$ However, other non-deconvolution-based methods exist and a formal comparison of the various methods has not yet been performed. ${ }^{5,50}$ The exact method to derive these parameters varies between vendors. In the deconvolution method, a point is chosen for the arterial input function and another point for the venous outflow function. Typically the A2 segment is selected for the arterial input function and the posterior superior sagittal sinus is selected for venous outflow, although this is also a debated issue. ${ }^{49,51} \mathrm{~A}$ normal CTP scan is shown in Figure 4.

There is considerable debate as to which CTP parameters best depict infarcted tissue from surrounding ischemic penumbra. ${ }^{5}$ In one theoretical model, there is a proposed failure of autoregulation of cerebral perfusion in infarcted tissue. As a result, in infarcted regions the CBV is decreased because of failure of vasodilatation and lack of increased collateral flow. Correspondingly, CBF is decreased and MTT is increased, while in ischemic penumbral tissue autoregulation is still intact, which results in vasodilatation and recruitment of collaterals to compensate for the decreased CBF. As a result, the CBV is normal or increased while the CBF is decreased and correspondingly the MTT is increased. As a practical matter, since gray and white matter CBF values at baseline differ significantly $(70 \mathrm{ml} / \mathrm{minute} / 100 \mathrm{~g}$ in gray matter compared with $20 \mathrm{ml} / \mathrm{minute} / 100 \mathrm{~g}$ in white matter) but MTT values between gray and white matter are not as variable (five seconds for gray matter and six seconds for white matter), MTT images are more sensitive to detect ischemic changes from baseline perfusion. ${ }^{52-54}$

An alternative hypothesis that results in similar changes in CBV, CBF, and MTT in the infarct core and penumbra implies the changes are primarily secondary to venous collapse in the infarcted tissue versus venous dilation in the penumbral tissue. ${ }^{3}$ Wintermark et al. confirmed these predicted changes in CTP parameters in one of the largest trials, evaluating data from 130 patients using receiver operating characteristic (ROC) curve analysis. They found that areas defined by an absolute CBV less than $2.0 \mathrm{ml} / 100 \mathrm{~g}$ best depicted the infarct core while regions defined by a relative MTT increase of $145 \%$ compared with the contralateral normal side best depicted the ischemic penumbra. Since there are known differences between vendors in the processing of CTP data, the use of absolute thresholds is of limited value for generalization purposes. As such, current expert opinion favors the use of relative CTP values. ${ }^{50}$ In this regard, the evaluation of infarct core proposed by Wintermark et al. may be best approximated by CBV values decreased by $65 \%$ relative to the normal contralateral side. ${ }^{52}$ Other proposed methods to depict infarct core from penumbra include absolute and relative CBF thresholds and CBF $\times$ CBV thresholds. ${ }^{55-58}$

At our institution, in the management of acute stroke patients we find colorimetric CTP parameter maps adequate for clinical
Figure 5: Examples of Compued Tomography Angiography and CT Perfusion to Evaluate Acute Stroke

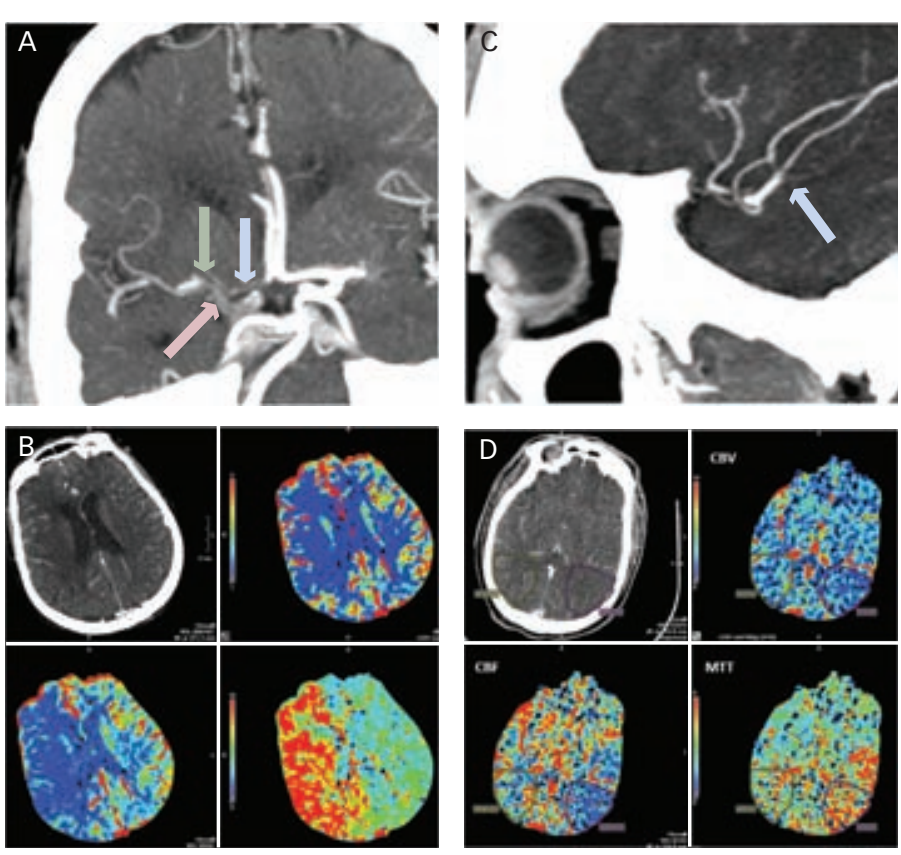

A: Computed tomography angiography (CTA) coronal multiplanar maximum-intensity projection (MIP) demonstrates a patient with a T-shaped internal carotid artery (ICA) occlusion involving the supra-clinoid ICA (red arrow), right M1 (green arrow), and right A1 (blue arrow). B: CT perfusion (CTP) scan of the patient demonstrates decreased cerebral blood volume (CBV) and cerebral blood flow (CBF) with prolonged mean transit time (MTT) in the right middle cerebral artery (MCA) territory consistent with infarction. There is prolonged MTT in the right ACA territory but with preserved $C B F$, consistent with decreased perfusion without completed infarction. C: CTA sagittal MIP demonstrates an inferior left M2 branch occlusion (blue arrow). $D$ : CTP demonstrated through the use of region of interest (ROI) quantitative measurements shows the CBV is approximately $70 \%$ of the normal contralateral side, suggesting severe ischemia/early acute infarct. The MTT abnormality is larger, indicating the presence of some ischemic penumbra.

decision-making. The perfusion images are always interpreted in the context of the clinical presentation and the vascular lesion based on the CTA.

Mismatch is determined by comparing the decreased CBV lesion with the prolonged MTT lesion by visual inspection. A typical case is shown in Figures $5 A$ and $5 B$. In select cases where the ischemic lesion is small, selective regions of interest (ROI) are drawn to perform a more quantitative analysis for confirmation, as shown in Figures $5 C$ and $5 D$. In the future, the analyses may become more automated, allowing for standardized post-processing and interpretation. ${ }^{59}$

One of the main limitations of CTP currently is its limited coverage. Most CTP protocols are centered at the basal ganglia and focus on coverage of the MCA territories. This is particularly problematic if the patient has posterior circulation ischemia, which may be missed because it was not imaged. Preliminary work with 256-channel MDCT demonstrates the feasibility of whole-brain CTP with associated radiation dose reduction as well. ${ }^{60}$ Another limitation of CTP is its limited ability to detect small infarcts. This can be problematic as small infarcts in eloquent locations can result in significant neurologic deficits. Additionally, detection of acute ischemia superimposed on chronic infarct or severe periventricular white matter ischemic changes can be difficult..$^{51}$ Lastly, CTP interpretation can be limited 
when there are bilateral vascular lesions, making comparison with the contralateral side difficult. In this situation the use of absolute thresholds may be useful.

\section{Conclusion}

Multimodal CT for the evaluation of acute stroke is becoming increasingly important as there continues to be a growing need to rapidly diagnose acute stroke patients. It is an ideal tool for imaging acute stroke patients in the community given its widespread availability and its rapid image acquisition. Multimodal CT demonstrates good sensitivity and specificity in diagnosing acute stroke.

There is also hope that the CTP component of multimodal CT will be able to discriminate among acute stroke patients by determining those who will likely benefit from intervention from those who would not regardless of their time of presentation. Considerable work still needs to be undertaken in this regard to standardize the processing and interpretation of CTP images.

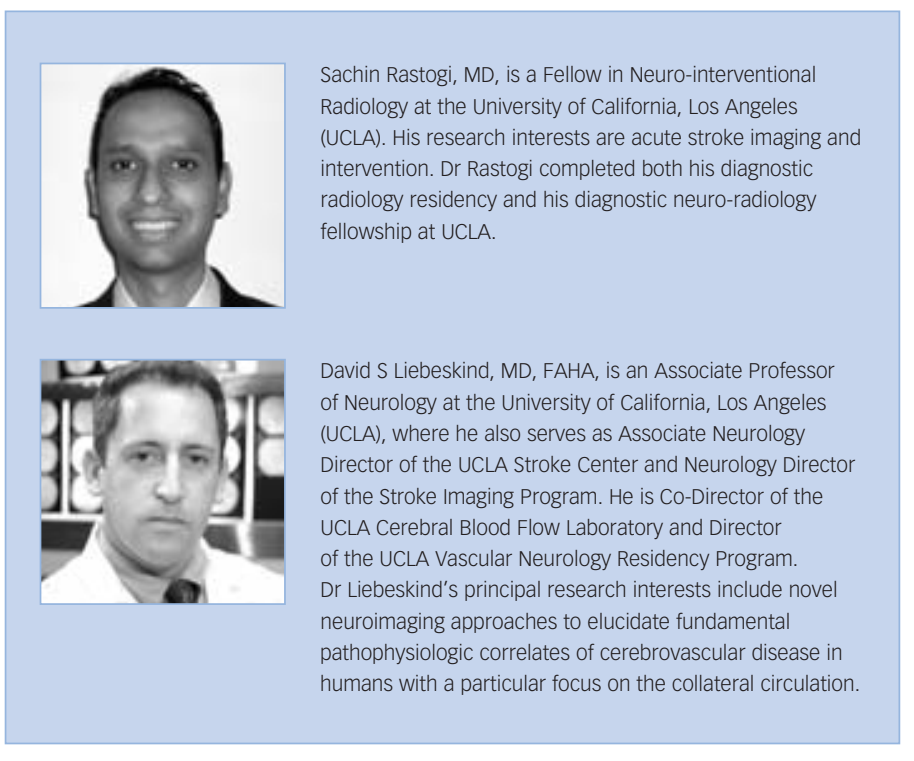

1. Lloyd-Jones D, Adams RJ, Brown TM, et al., Circulation, 2010;121(7):948-54.

2. Schumacher HC, Bateman BT, Boden-Albala B, et al., Ann Emerg Med, 2007;50(2):99-107.

3. Liebeskind DS, Ann Neurol, 2009;66(5):574-90.

4. Shetty SK, Lev MH, Neuroimaging Clin N Am Aug, 2005;15(3):481-501, ix

5. Wintermark $\mathrm{M}$, Albers GW, Alexandrov AV, et al., AJNR Am Neuroradiol, 2008;29(5):e23-30.

6. Aspelin P, Aubry P, Fransson SG, et al., N Eng/ J Med, 2003;348(6):491-9.

7. Hernandez F, Mora L, Garcia-Tejada J, et al., Rev Esp Cardiol, 2009;62(12):1373-80.

8. Srinivasan A, Goyal M, Lum C, et al., Can J Neurol SCi, 2005;32(4):483-6.

9. Beauchamp NJ Jr, Barker PB, Wang PY, van Zijl PC, Radiology, 1999;212(2):307-24.

10. Wardlaw JM, Mielke O, Radiology, 2005;235(2):444-53.

11. von Kummer R, Bourquain H, Bastianello S, et al., Radiology, 2001;219(1):95-100.

12. Lev MH, Farkas J, Gemmete JJ, et al., Radiology, 1999;213(1):150-55.

13. Gonzalez RG, Hirsch JA, Koroshetz WJ, et al., Acute Ischemic Stroke: Imaging and Intervention, New York: Springer, 2006.

14. Barber PA, Demchuk AM, Hudon ME, et al., Stroke, 2001;32(1):84-8

15. Mullins ME, Semin Ultrasound CT MR, 2005;26(6):394-403

16. Tomsick T, Brott T, Barsan W, et al., AJNR Am J Neuroradiol, 1992;13(1):257-63.

17. von Kummer $\mathrm{R}$, Allen $\mathrm{KL}$, Holle $\mathrm{R}$, et al., Radiology, 1997;205(2):327-33.

18. von Kummer R, Meyding-Lamade $\mathrm{U}$, Forsting $\mathrm{M}$, et al., AJNR Am J Neuroradiol, 1994;15(1):9-15, discussion 16-18.

19. Adams HP, Jr, Brott TG, Furlan AJ, et al., Circulation, 1996:94(5):1167-74

20. The NINDS t-PA Stroke Study Group, Stroke, 1997:28(11):2109-18

21. Launes J, Ketonen L, J Neurol Neurosurg Psychiatry,
1987:50(11):1550-52.

22. Hacke W, Kaste M, Fieschi C, et al., JAMA, 1995:274(13):1017-25.

23. Tanne D, Kasner SE, Demchuk AM, et al., Circulation, 2002:105(14):1679-85.

24. Barber PA, Demchuk AM, Zhang J, Buchan AM, Lancet, 2000;355(9216):1670-74.

25. de Weert TT, Cakir H, Rozie S, et al., AJNR Am J Neuroradiol 2009;30(1):177-84.

26. Otani $\mathrm{H}$, Kikuya $\mathrm{M}$, Hara $\mathrm{A}$, et al., Cerebrovasc Dis, 2010;30(1):43-50

27. Menon U, Kelley RE, Int Rev Neurobiol, 2009;84:21-33.

28. Hsu LC, Hu HH, Chang CC, et al., Zhonghua Yi Xue Za Zhi (Taipei) 1997:59(4):225-31.

29. Bischoff C, von Einsiedel HG, Nervenarzt, 1997;68(8):609-19.

30. Chen XY, Lam WW, Ng HK, et al., Cerebrovasc Dis, 2006;21

(1-2):91-7.

31. Forsting M, Eur Radiol, 2005;15(Suppl. 4):D25-7.

32. McKinney AM, Palmer CS, Truwit CL, et al., AJNR Am 」 Neuroradiol, 2008;29(3):594-602.

33. Lev MH, Farkas J, Rodriguez VR, et al., J Comput Assist Tomogr, 2001;25(4):520-28.

34. Sims JR, Rordorf G, Smith EE, et al., AJNR Am J Neuroradiol, 2005;26(2):246-51.

35. Wildermuth $\mathrm{S}$, Knauth $\mathrm{M}$, Brandt $\mathrm{T}$, et al., Stroke, 1998;29(5):935-8.

36. Sen S, Huang DY, Akhavan O, et al., Neurocrit Care, 2009:11(1):76-81

37. Gleason S, Furie KL, Lev MH, et al., Acad Radiol, 2001;8(10):955-64.

38. Puchner S, Popovic M, Wolf F, et al., J Endovasc Ther, 2009;16(3):336-42

39. Wintermark M, Jawadi SS, Rapp JH, et al., AJNR Am J Neuroradiol, 2008;29(5):875-82.

40. Bartlett ES, Walters TD, Symons SP, Fox AJ, AJNR Am J Neuroradiol, 2006;27(3):632-7.

41. Taschner CA, Leclerc $X$, Lucas $C$, Pruvo JP, Front Neurol Neurosci, 2005;20:119-28.
42. Oelerich M, Stogbauer F, Kurlemann G, Schul C, Schuierer G, Eur Radiol, 1999;9(7):1385-91.

43. Puetz V, Sylaja PN, Coutts SB, et al., Stroke, 2008;39(9):2485-90.

44. Puetz V, Sylaja PN, Hill MD, et al., AJNR Am I Neuroradiol, 2009;30(10):1877-83.

45. Schramm P, Schellinger PD, Klotz E, et al., Stroke, 2004;35(7):1652-8

46. Schramm P, Schellinger PD, Fiebach JB, et al., Stroke, 2002;33(10):2426-32.

47. Kloska SP, Nabavi DG, Gaus C, et al., Radiology, 2004:233(1):79-86.

48. Knoepfli AS, Sekoranja L, Bonvin C, et al., J Neuroradiol, 2009:36(3):131-7.

49. Konstas AA, Goldmakher GV, Lee TY, Lev MH, AJNR Am 」 Neuroradiol, 2009;30(4):662-8.

50. Konstas AA, Goldmakher GV, Lee TY, Lev MH, AJNR Am J Neuroradiol, 2009;30(5):885-92

51. Lui YW, Tang ER, Allmendinger AM, Spektor V, AJNR Am 」 Neuroradiol, 2010 (Epub ahead of print)

52. Wintermark $M$, Flanders $A E$, Velthuis $B$, et al., Stroke, 2006;37(4):979-85

53. Wintermark M, Reichhart M, Cuisenaire O, et al., Stroke, 2002;33(8):2025-31.

54. Wintermark M, Fischbein NJ, Smith WS, et al., AJNR Am J Neuroradiol, 2005:26(1):104-12.

55. Eastwood JD, Lev MH, Azhari T, et al., Radiology, 2002;222(1):227-36.

56. Murphy BD, Fox AJ, Lee DH, et al., Stroke, 2006;37(7):1771-7.

57. Eastwood JD, Lev MH, Wintermark $\mathrm{M}$, et al., AJNR Am 」 Neuroradiol, 2003;24(9):1869-75.

58. Mayer TE, Hamann GF, Baranczyk J, et al. AJNR Am J Neuroradiol, 2000;21(8):1441-9.

59. Goldmakher GV, Paper presented at: Radiological Society of North America Scientific Assembly and Annual Meeting Program, 2006; Oak Brook, III.

60. Mori S, Obata T, Nakajima N, et al., AJNR Am I Neuroradiol, 2005;26(10):2536-41. 